\title{
Specific functionalization of CTAB stabilized anisotropic gold nanoparticles with polypeptides for folding-mediated self-assembly
}

\author{
Yusong Wang, Daniel Aili, Robert Selegård, Yeeyan Tay, Lars Baltzer, \\ Hua Zhang and Bo Liedberg
}

\section{Linköping University Post Print}

N.B.: When citing this work, cite the original article.

Original Publication:

Yusong Wang, Daniel Aili, Robert Selegård, Yeeyan Tay, Lars Baltzer, Hua Zhang and Bo Liedberg, Specific functionalization of CTAB stabilized anisotropic gold nanoparticles with polypeptides for folding-mediated self-assembly, 2012, Journal of Materials Chemistry, (22), 38, 20368-20373.

http://dx.doi.org/10.1039/c2jm31176c

Copyright: Royal Society of Chemistry http://www.rsc.org/

Postprint available at: Linköping University Electronic Press http://urn.kb.se/resolve?urn=urn:nbn:se:liu:diva-84914 


\title{
Specific functionalization of CTAB stabilized anisotropic gold nanoparticles with polypeptides for folding-mediated self-assembly ${ }^{\dagger}$
}

\author{
Yusong Wang, ${ }^{a, b}$ Daniel Aili, ${ }^{a, c}$ Robert Selegård, ${ }^{c}$ Yeeyan Tay, ${ }^{b}$ Lars Baltzer, ${ }^{d}$ Hua Zhang, ${ }^{a, b}$ Bo \\ Liedberg*a,c
}

\author{
s Received (in $X X X, X X X)$ Xth $X X X X X X X X X 20 X X$, Accepted Xth $X X X X X X X X X 20 X X$ \\ DOI: $10.1039 / b 000000 x$
}

Anisotropic nanoparticles stabilized by cetyltrimethylammonium bromide (CTAB) are notoriously

difficult to homogenously functionalize using conventional gold-thiol chemistry. Using surface assisted

laser desorption time of flight mass spectroscopy and scanning transmission electron microscopy-energy

10 dispersive X-ray spectroscopy, we demonstrate that silver species adsorbed on the particle surface prevent effective surface functionalization. When covered by a thin gold film, particle functionalization was drastically improved. A thiol-containing polypeptide was immobilized on arrowhead gold nanorods (NRs) and was subsequently able to selectively heteroassociate with a complementary polypeptide resulting in a folding-mediated bridging aggregation of the NRs. Despite using arrowhead NRs with a ${ }_{15}$ pronounced difference in surface arrangement on the $\{111\}$ facets on the arrowheads compared to the $\{100\}$ facets at the particle sides, the polypeptides were efficiently and homogeneously immobilized on the particles after gold film overgrowth.

\section{Introduction}

Controllable self-assembly of plasmonic metal nanoparticles is of 20 large interest for the development of novel diagnostic and therapeutic tools, and for applications and fundamental studies of plasmon enhanced phenomena such as surface enhanced Raman scattering. Gold nanoparticles (Au NPs) and anisotropic Au NPs in particular, have unique optical properties making them highly 25 interesting for these purposes. The utilization of biomolecules to tune the surface properties and assembly of Au NPs is a very attractive approach that has received considerable attention. A range of different biomolecules have been examined for functionalization of $\mathrm{Au} \mathrm{NPs}$, including DNA, ${ }^{[1]}$ aptamers, ${ }^{[2]}$ 30 proteins $^{[3]}$ and polypeptides. ${ }^{[4]}$ In parallel, there has been a rapid progress in the development of synthesis routs for obtaining $\mathrm{Au}$ NPs with various shapes and sizes. A commonly employed method for synthesis of rod-shaped Au NPs involves the use of the surfactant cetyltrimethylammonium bromide (CTAB) in 35 combination with small amounts of silver ions. CTAB forms densely packed bilayers on the nanoparticle surface and is critical for obtaining non-spherical particles, whereas the presence of silver ions has a dramatic effect on particle shape and crystallinity. The presence of silver ions also increases the yield 40 of rod-shaped particles. ${ }^{[5]}$

Although necessary for their synthesis and stability in suspension, the presence of CTAB and silver ions significantly complicates further surface functionalization through conventional gold-thiol chemistry. Difficulties with tailoring the 45 surface chemistry of CTAB capped nanoparticles without compromising particle stability has significantly limited their applicability in analytical devices. CTAB is also highly cytotoxic $^{[6]}$ which has prevented widespread use of CTAB capped nanoparticles in therapeutic applications. Numerous strategies for

50 functionalizing CTAB stabilized particles in order to render them biocompatible and chemically versatile have been proposed, including physisorption of polymers. ${ }^{[7]}$ In a recent paper, Mirkin and co-workers demonstrated that by growing a thin gold film around CTAB stabilized gold nanorods (Au NRs) after synthesis, 55 the particles could effectively be functionalized with thiolated single-stranded DNA. ${ }^{[8]}$ They further hypothesized that the difficulty in immobilizing the DNA before the overgrowth procedure was due to the presence of silver at the nanoparticle surface that prevented the formation of a stable Au-S bond.

60 The physicochemical role of silver on controlling the shape and properties of anisotropic nanoparticles as well as the nature of the silver species present on the nanoparticle surface is still under debate. Silver ions have been proposed to react with CTAB to form silver bromide or a CTAB-silver bromide complex, 65 which preferentially binds to the $\{100\}$ and $\{110\}$ facets of the rod thus affecting the anisotropic growth. ${ }^{[9]}$ Moreover, silver ions have been suggested to form a monolayer or submonlayer of $\operatorname{Ag}(0)$ on the particles by underpotential deposition of $\operatorname{Ag}(\mathrm{I})$ at the $\{110\}$ and $\{100\}$ facets during the growths process, with similar 70 results. ${ }^{[10]}$ Using Surface Assisted Laser Desorption-Time of Flight Mass Spectroscopy (SALDI-TOF MS), Nakashima and coworkers identified $\mathrm{AgBr}^{2-}$ adsorbed on the particle surface as the key component influencing particle shape. ${ }^{[11]}$ Silver is thus most likely present in several forms on the particle surface and 75 are, in addition to the presence of CTAB, probably responsible for the difficulties with surface functionalization. 
Here, we show that the surface concentration of silver species on arrowhead Au NRs with well-defined $\{111\}$ facets plays a critical role on the functionalization of the particles. The presence and relative concentration of silver on the particle surface before 5 and after overgrowth of the particles with a thin gold film were determined using SALDI-TOF MS in combination with Scanning Transmission Electron Microscopy-Energy Dispersive X-ray Spectroscopy (STEM-EDS). We further demonstrate that particles with an additional gold layer can be specifically and 10 homogenously functionalized with thiol-containing molecules. A well characterized set of helix-loop-helix polypeptides that were de novo designed to assemble into heterodimeric four-helix bundles were used to demonstrate the role of the additional gold film. ${ }^{[12]}$ The possibility to induce and tune the heteroassociation 15 and folding of the polypeptides into four-helix bundles enabled highly controllable self-assembly of the particles.

By establishing new strategies for tuning the surface chemistry of anisotropic gold nanoparticle, their applicability will increase dramatically. The specific folding-mediated particle assembly 20 demonstrated here, in combination with the unique optical properties of the arrowhead Au NRs, is of large interest for the development of novel bioanalytical devices and assays based on e.g. surface enhanced Raman scattering and metal enhanced fluorescence.

\section{${ }_{25}$ 2. Experimental Section}

\section{Chemicals}

Cetyltrimethylammonium bromide (CTAB, $\geq 96 \%$ ), sodium borohydride $\left(\mathrm{NaBH}_{4}, 99.99 \%\right)$, L-ascorbic acid (AA, 99\%+), hydrogen tetrachloroaurate(III) trihydrate $\left(\mathrm{HAuCl}_{4} \cdot 3 \mathrm{H}_{2} \mathrm{O}, \geq\right.$ 30 99.9\%), and silver nitrate $\left(\mathrm{AgNO}_{3}, 99.9999 \%\right)$ were all purchased from Sigma-Aldrich. Milli-Q water (18.2 M 2 ) was used in all experiments. The synthesis of the polypeptides (JR2KC, JR2EC, JR2ECref) is described in detail elsewhere. ${ }^{[12 b]}$ Peptide sequences can be found in the supporting information. Briefly, the 35 polypeptides were generated on a Pioneer automated peptide synthesizer using standard Fmoc chemistry. Following synthesis, the peptides were cleaved, precipitated, HPLC purified and identified by MALDI-TOF mass spectrometry.

In order to obtain the reduced polypeptides $\mathrm{JR}_{2} \mathrm{EC}_{2}$ and ${ }_{40} \mathrm{JR}_{2 \mathrm{ECref}}$, JR2EC and JR2ECref $(1 \mathrm{mM}, 25 \mu \mathrm{L})$ were dissolved in sodium bicarbonate solution $(225 \mu \mathrm{L}, 0.1 \mathrm{M}, \mathrm{PH}=8.3)$. The solution was purged with oxygen for $90 \mathrm{~min}$ followed by incubation at $4{ }^{\circ} \mathrm{C}$ for at least 24 hours before use. The complete oxidation to was confirmed by a standard Ellman's test for 45 determination of free thiols. ${ }^{[16]}$

\section{Synthesis of gold nanorods}

CTAB-stabilized gold nanorods (NRs) were synthesized by a slightly modified seed-mediated growth method. ${ }^{[5 b, 13,17]}$ Briefly, 50 the seed solution was prepared by the addition of a freshly prepared, ice-cold aqueous $\mathrm{NaBH}_{4}$ solution $(0.01 \mathrm{M}, 0.6 \mathrm{~mL})$ into an aqueous mixture composed of $\mathrm{HAuCl}_{4}(0.01 \mathrm{M}, 0.25 \mathrm{~mL})$ and CTAB (0.1 M, $7.5 \mathrm{~mL})$. The resultant solution was mixed by rapid inversion for $2 \mathrm{~min}$ and then kept at room temperature for ${ }_{55} \sim 1 \mathrm{~h}$ before use. The Au NR growth solution was made by first mixing aqueous solutions of CTAB $(0.1 \mathrm{M}, 40 \mathrm{~mL}), \mathrm{HAuCl}_{4}$ (0.01 M, $2 \mathrm{~mL}), \mathrm{H}_{2} \mathrm{SO}_{4}(0.8 \mathrm{~mL}, 0.5 \mathrm{M})$, and $\mathrm{AgNO}_{3}(0.01 \mathrm{M}$,
$0.4 \mathrm{~mL})$. After gently mixing the solution, a freshly prepared aqueous ascorbic acid solution $(0.1 \mathrm{M}, 0.32 \mathrm{~mL})$ was added. ${ }_{60}$ After the resultant solution was homogenized by gentle inversion and became colorless, the seed solution $(0.096 \mathrm{~mL})$ was then added into the reaction mixture with gentle inversion for $\sim 20 \mathrm{~s}$, and then left undisturbed for $\sim 14 \mathrm{~h}$ at room temperature. Excess CTAB was removed by centrifugation and supernatant removal. ${ }_{65}$ The precipitated nanorods were redispersed in $10 \mathrm{~mL}$ Milli-Q water with $\sim 5 \mathrm{mM} \mathrm{CTAB}$ for further use.

\section{Synthesis of gold arrowhead nanorods}

The synthesis of the $\mathrm{Au}$ arrowhead NRs were carried out 70 according to a slightly modified procedure. ${ }^{[13]}$ In a typical synthesis of the Au arrowhead NRs, $0.5 \mathrm{~mL}$ as-prepared Au NRs were mixed with aqueous solutions of CTAB $(2.0 \mathrm{~mL}, 0.20 \mathrm{M})$, ascorbic acid $(240 \mu \mathrm{L}, 0.788 \mathrm{M})$, and $\mathrm{AgNO}_{3}(240 \mu \mathrm{L}, 4.0 \mathrm{mM})$ respectively, followed by the addition of $\mathrm{HAuCl}_{4}(64 \mu \mathrm{L}, 15 \mathrm{mM})$ 75 solution. The mixture was then diluted with Milli-Q water to give a final volume of $12 \mathrm{~mL}$ and incubated in a water bath at $30^{\circ} \mathrm{C}$ for at least 12 hours. Then, the reaction product was centrifuged at room temperature, and the supernatant was removed and the NRs were redispersed in $2 \mathrm{~mL}$ Milli-Q water with $\sim 5 \mathrm{mM} \mathrm{CTAB}$ 80 for further use.

For overgrowth of the NRs with an additional gold layer, 0.2 $\mathrm{mL}$ of the NRs were dispersed in $0.8 \mathrm{~mL}$ Milli-Q water. After centrifugation and removal of the supernatant, the NRs were dispersed in CTAB solution (490 uL, 0.01M). Ascorbic acid (10 $85 \mu \mathrm{L}, 100 \mathrm{mM})$ and $\mathrm{HAuCl}_{4}(15 \mathrm{mM})$ were then added into the above solution and after allowing $\sim 1$ hour overgrowth, the solution was brought to $0.05 \mathrm{M} \mathrm{CTAB}$ and incubated for $\sim 30$ mins. The final concentration of $\mathrm{HAuCl}_{4}$ added was varied from 0 $\mu \mathrm{M}$ to $386 \mu \mathrm{M}$. The modified particles were centrifuged twice 90 and the supernatant was replaced with Milli-Q water and finally redispersed in $135 \mu \mathrm{L}$ acetate buffer $(64 \mathrm{mM}, \mathrm{pH}=5.5)$.

\section{Nanorod functionalization}

For immobilization of JR2KC on $\mathrm{Au}$ arrowhead NRs, the ${ }_{95}$ particles were mixed with JR2KC dissolved in MQ water $(15 \mu \mathrm{L}$, $1 \mathrm{mM}$ ) while stirring. The resulting reaction mixture was vortexed briefly and incubated for at least 3 hours at room temperature. A $\mathrm{NaCl}(16.67 \mathrm{uL}, 5 \mathrm{M})$ solution was gradually added into the above solution during 1.5 hours with $30 \mathrm{~min}$ 100 interval and left to incubate for 2 days at room temperature. The particles were subsequently washed with buffer $(10 \mathrm{mM}$ acetate buffer $\mathrm{pH} 5.5$ with $1 \mathrm{mM}$ CTAB) by repeated centrifugation and supernatant removal until the concentration of unbound peptide was $<1 \mathrm{nM}$, and finally redispersed in $75 \mu \mathrm{L}$ buffer.

105

\section{Peptide folding-induced assembly of NRs}

The JR2KC functionalized NRs $(3 \mu \mathrm{L})$ were dispersed in HBSEP buffer $([\mathrm{Au} \mathrm{NR}]=\sim 0.1 \mathrm{nM})$. JR2EC or JR2ECref $_{2}(50 \mu \mathrm{M}$, $3.7 \mu \mathrm{L}$ ) was added into the above solution and mixed well. UV110 vis was used to monitor the spectrum changes at 2-minute intervals. $\mathrm{pH}$-mediated $\mathrm{NR}$ assembly and disassembly was induced by addition of $\mathrm{NaOH}$ and $\mathrm{HCl}$, respectively.

\section{Instrumentation and characterization}

${ }_{115}$ UV-vis spectroscopy (Shimadzu 1800) was used to collect 
extinction spectra of NRs with the scanning range from $900 \mathrm{~nm}$ to $400 \mathrm{~nm}$. Circular dichroism spectra were recorded with a CD6 Spectrodichrograph (JobinYvon-Spex) using a $0.1 \mathrm{~mm}$ cuvette at room temperature. Each spectrum was collected as an average of 5 three scans in the range $190-260 \mathrm{~nm}$. The high-resolution TEM (HR-TEM) images were obtained using a JEOL model JEM-2010 or TEM-2100F microscope operating at $200 \mathrm{kV}$. EDS line profiling was conducted under STEM-BF mode (TEM-2100F). Electron beam spot size: $1 \mathrm{~nm}$, camera length: $20 \mathrm{~cm}$, and dwell 10 time: $3000 \mathrm{~ms}$. The DT\% is maintained $\sim 20 \%$ and CPS is $\sim 1000$. Drift function was enabled to track the electron beam spot during STEM-EDS line profiling along a single AHNR. The field emission scanning electron microscopy (FE-SEM 7600F, $15 \mathrm{kV}$ ) was also used to characterize the aggregation status of NRs in 15 TED mode. The suspensions were incubated on the TEM copper grids for 2 minutes before being wicked off by a filter paper to remove excess solution and dried in vacuum.

Surface assisted laser desorption and ionization-time of flightmass spectrometer (SALDI-TOF-MS) were conducted using a 20 Axima TOF $^{2}$ mass spectrometry (Shimazu/Kratos, Singapore) equipped with a nitrogen laser at $337 \mathrm{~nm}$ (Pulse $10 \mathrm{~ns}, 10 \mathrm{~Hz}$ ) for ionization/desorption of analytes. Each spectrum was recorded by adding 100 laser shots using a laser energy of $43.44 \mu \mathrm{J}$ (Instrumental parameter: 65 in the scale of 180). The accelerating 25 voltage was $20 \mathrm{kV}$, and the operating pressure was $10^{-7}$ mbar. All the mass spectra shown in the paper were obtained in a negative ion mode.

\section{Results and Discussion}

\subsection{Nanoparticle synthesis and characterization}

30 Arrowhead Au NRs were prepared by a seed-mediated synthesis of cylindrical NRs, followed by the synthesis of two arrowhead ends. The overgrowth of the NRs with gold films of increasing thickness was accomplished by varying the concentration of the gold salt from 0-360 $\mu \mathrm{M}$. The gold was reduced with ascorbic 35 acid in the absence of silver nitrate. SALDI-TOF was utilized to investigate the relative abundance of anionic species at the surface of the particles before and after the overgrowth procedure. The results show that $\mathrm{AgBr}_{2}^{-}(\mathrm{m} / \mathrm{z}=266.6)$ and $\mathrm{AuBr}_{2}^{-}(\mathrm{m} / \mathrm{z}=356.6)$ were the two major species along with 40 bromide $(\mathrm{m} / \mathrm{z}=78.9)$ in all samples (Figure $1 \mathrm{a}, 1 \mathrm{~b}$ ), which is similar to previously reported data on NRs. ${ }^{[11]} \mathrm{AgBr}^{2-}$ was present at the particle surface both before and after the overgrowth procedure but the $\left(\mathrm{AuBr}^{2-}\right):\left(\mathrm{AgBr}^{2-}\right)$ peak intensity ratio, however, increased linearly with increasing concentration of ${ }_{45} \mathrm{HAuCl}_{4}$ used during the overgrowth process (Figure 1c). This suggests that by this procedure the silver ions at the particle surface were gradually covered or replaced by the gold. The overgrowth was also accompanied by a blue-shift of the longitudinal plasmon band from about $710 \mathrm{~nm}$ for the untreated ${ }_{50} \mathrm{NRs}$ to about $630 \mathrm{~nm}$ for particles with the thickest gold film (386 $\mu \mathrm{M} \mathrm{HAuCl}_{4}$ during the growth) (Figure 1c). Such a blue shift of LSPR band follows the growth pattern of arrowhead $\mathrm{Au}$ NRs during synthesis, ${ }^{[13]}$ indicating that the gold film thickness increase with increasing concentration of the gold salt. The actual

55 thickness of the gold film could not be determined here using high-resolution electron micrographs

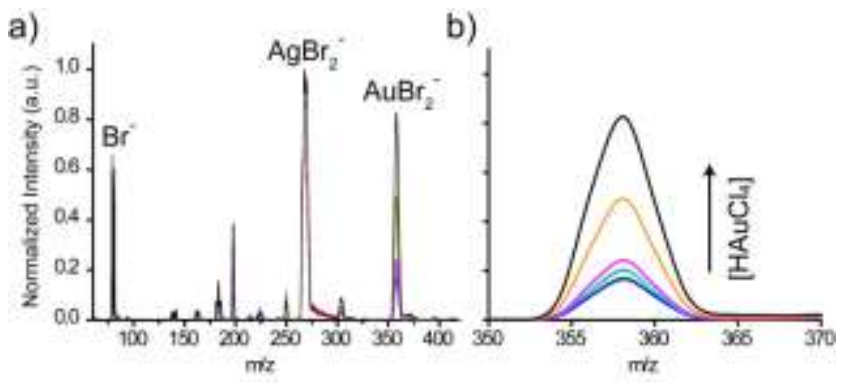

c)

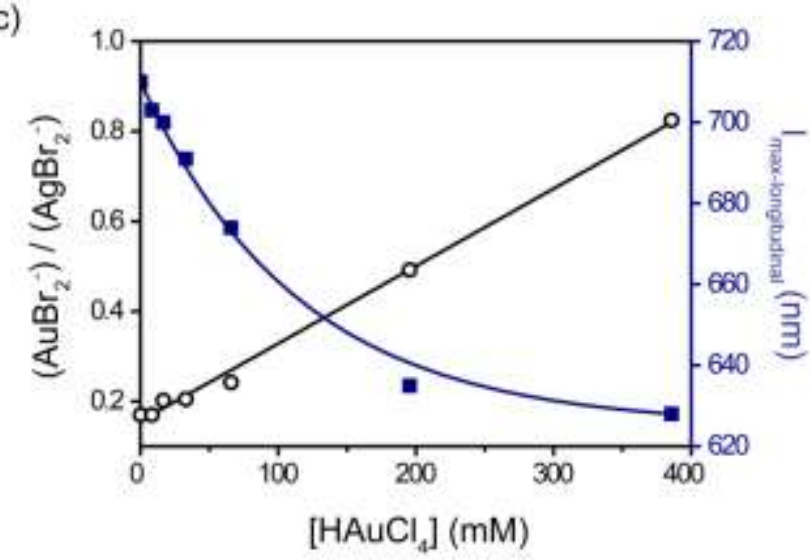

Figure 1. a) SALDI-TOF spectra of Au AHNR subjected to a gold overgrowth at increasing concentrations of $\mathrm{HAuCl}_{4}$. b) Magnified view of 60 the $\mathrm{AuBr}_{2}$ peak. Spectra are normalized with respect to the $\mathrm{AgBr}_{2}$ peak. c) The dependence of $\mathrm{I}\left(\mathrm{AuBr}_{2}^{-}\right) / \mathrm{I}\left(\mathrm{AgBr}_{2}^{-}\right)$on the $\left[\mathrm{HAuCl}_{4}\right]$ used during the gold layer growth (circles) and the corresponding longitudinal LSPR peak position of the Au AHNRs (squares).
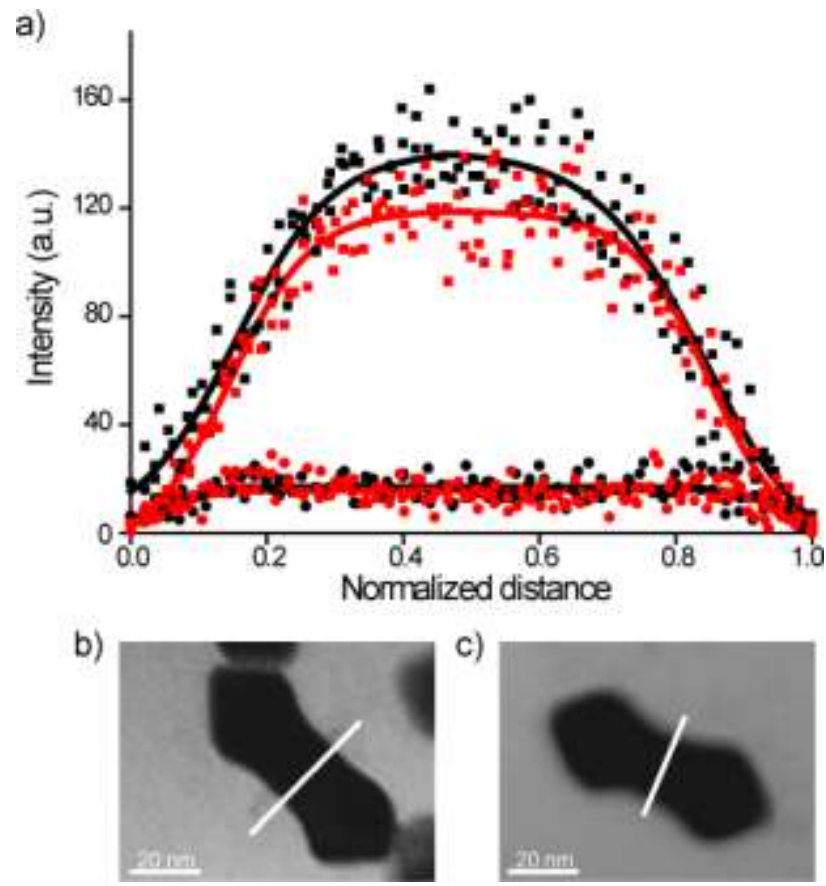

Figure 2. a) STEM-BF EDS line profiling along the cross-section of the $\mathrm{NRs}$ before (red) and after (black) overgrowth using a $\mathrm{HAuCl}_{4}$ concentration of $16.45 \mu \mathrm{M}$. Gold: squares, Silver: circles. Data from three different particles of each sample are shown. Representative STEM-BF 70 images of the NRs before (b) and after (c) overgrowth, ([HAuCl $\left.{ }_{4}\right]=16.45$ $\mu \mathrm{M}$ during Au layer growth). White lines indicate the EDS profiling position during STEM-BF EDS 
(Figure S1a and Figure S1b, Supporting Information).

STEM-EDS further confirmed that the gold overgrowth covers the silver species rather than removing them from the particle surface (Figure 2) since the intensity of the Au signal increased 5 after the overgrowth while the Ag signal remained unchanged. The Au:Ag intensity ratio increased from $\sim 6.0$ to $\sim 7.2$ for the unmodified particles and particles with a thin gold film $\left(\mathrm{HAuCl}_{4}\right.$ concentration of $16.45 \mu \mathrm{M}$ during the growth process), respectively.

10

\subsection{Peptide Functionalization}

The 42 amino acid helix-loop-helix polypeptides JR2KC and JR2EC are de novo designed to heterodimerize and fold into fourhelix bundles. ${ }^{[12]}$ The driving force for folding is the formation of 15 a hydrophobic core formed by the amphipathic helices. A relatively large number of charged residues, predominately Lys in JR2KC and Glu in JR2EC, at the dimer interface prevents homodimerization at neutral $\mathrm{pH}$. Homodimerization occurs under basic and acidic conditions for JR2KC and JR2EC, respectively, 20 as a result of charge neutralization. A single Cys residue in the loop region enables directed thiol-mediated immobilization on gold but can also be utilized to covalently link two monomers by a disulphide bridge. When oxidized, these peptides self-assemble into fibrous nanostructures by a folding-mediated 25 heteroassociation process. ${ }^{[14]}$ Immobilization of the Glu rich polypeptide JR2EC on spherical gold nanoparticles yields highly stable dispersions. Addition of oxidized JR2KC $\left(\mathrm{JR} 2 \mathrm{KC}_{2}\right)$ to JR2EC modified particles results in a folding dependent bridging aggregation, caused by the heteroassociation and folding of the 30 peptides into disulphide-linked four-helix bundles that span in between the particles. ${ }^{[12 b]}$ Here, the immobilization of JR2KC onto NRs before and after overgrowth of the NRs with a thin gold film was investigated and the particle assembly after addition of oxidized JR2EC (JR2EC 2 ) was characterized.

35 Immobilization of JR2KC on spherical, citrate capped Au NPs, results in an immediate particle aggregation, which most likely is caused by an unspecific bridging of the negatively charged particles by the positively charged polypeptides. Addition of JR2KC to the CTAB stabilized NRs prepared here did, however, 40 not affect the stability of the particles as they remained dispersed, indicating that the peptide were either not immobilized or unable to bridge the particles. After overnight incubation with JR2KC and removal of unbound peptides left in solution by repeated centrifugations, still no apparent changes in stability were 45 observed. Addition of the oxidized complementary peptide $\mathrm{JR}_{2} \mathrm{EC}_{2}$ caused a small decrease in the UV-vis extinction but no red-shift of the plasmon bands indicative of aggregation could be seen (Figure 2a). If, on the other hand, immobilizing JR2KC on particles modified with an additional thin gold film, a dramatic 50 difference was observed. The particles were highly stable in dispersion after incubation with the JR2KC peptide and during subsequent washing steps, but extensive aggregation was observed upon addition of $\mathrm{JR}_{2} \mathrm{EC}_{2}$ (Figure 2b), strongly indicating that JR2KC was immobilized on the particles and able 55 to heteroassociate with $\mathrm{JR}_{2} \mathrm{EC}_{2}$. The presence of the peptide on the nanoparticle surface was confirmed by FT-IR spectroscopy. The IR-spectrum showed two distinct bands corresponding to the amide I $\left(1655 \mathrm{~cm}^{-1}\right)$ and amide II $\left(1544 \mathrm{~cm}^{-1}\right)$, originating from vibrational modes in the polypeptide backbone (Figure S2, ${ }_{60}$ Supporting information).

NRs have two very distinct plasmon bands emanating from the transverse and longitudinal oscillations of the electrons. The aggregation of NRs has been
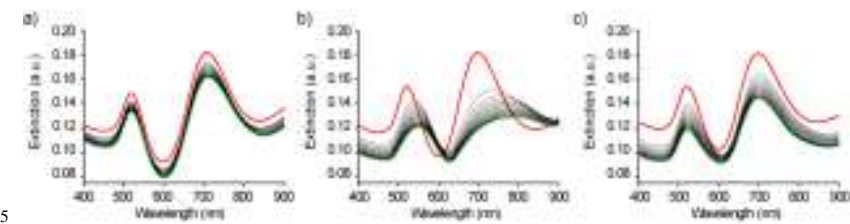

Figure 3. UV-vis extinction spectra recorded over 30 minutes of NRs $(\sim 0.1 \mathrm{nM})$ after functionalization with JR2KC before and after addition of $2.5 \mu \mathrm{M}$ of the complementary polypeptide JR2EC 2 . a) NRs without an additional gold layer and $\mathrm{b}$ ) NRs with an additional gold layer ([HAuCl$\left.{ }_{4}\right]$ $70=16.5 \mu \mathrm{M}$ during Au layer growth). $\mathrm{c}$ ) NRs (same as in b) after addition of a reference peptide JR2ECref ${ }_{2}$ in which all L-Ala has been replace with D-Ala.

reported to induce different responses in the plasmon bands 75 depending on the relative orientation of the particles. ${ }^{[15]}$ An endto-end orientation, resulting in a strong coupling of the longitudinal modes, produces a pronounced spectral red-shift of this band, whereas a side-to-side orientation results in a spectral blue-shift. Here, pronounced red-shifts of both the longitudinal 80 and the transverse plasmon bands were obtained (Figure 3), indicating random aggregation. The shift of the transverse plasmon peak reached equilibrium within 10 minutes after addition of the bridging polypeptide whereas the longitudinal peak continued to change even after 30 minutes (Figure 4a), 85 reflecting the difference in the sensitivity of the two plasmon bands towards changes in the polarizability of the vicinity of the particles as well as differences in their sensing depth. The particles eventually precipitated as the aggregation process proceeded.

90

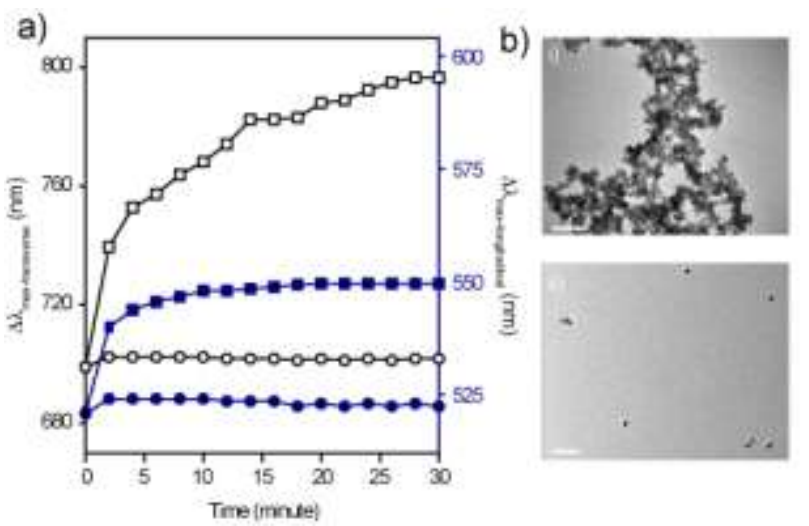

Figure 4. a) Change of the wavelength of maximum extinction over time or JR2 KC functionalized NRs $(\sim 0.1 \mathrm{nM})$ in the presence of $2.5 \mu \mathrm{M}$ $\mathrm{JR}_{2} \mathrm{EC}_{2}$ (squares) and $2.5 \mu \mathrm{M} \mathrm{JR} 2 \mathrm{ECref}_{2}$ (circles). The longitudinal 95 plasmon mode $\left(\lambda_{\text {max-longitudinal }}\right)$ is presented on the left y-axis (black) and the transverse plasmon mode ( $\left.\lambda_{\text {max-transverse }}\right)$ on the right y-axis (blue). b) Representative electron micrographs of corresponding particles after 30 minutes incubation with JR2EC 2 (top) and JR2 $\mathrm{ECref}_{2}$ (bottom).

100 Field emission scanning electron microscope (FESEM) using 
transmission electron detector (TED) imaging showed a gradual increase in aggregate size without any observable anisotropy in aggregate morphology (Figure S3, Supporting Information), indicating that the polypeptides were homogenously immobilized 5 on the particle surface despite the very pronounced different surface arrangement and surface energy between $\{111\}$ facets on the arrowheads and the $\{100\}$ at the side. ${ }^{[13]}$

In order to exclude unspecific bridging as a cause for the aggregation and to probe the influence of folding on the assembly 10 of the JR2KC-modified particles, a polypeptide with the identical amino acid sequence as $\mathrm{JR}_{2} \mathrm{EC}_{2}$ but where all L-Ala had been exchanged by D-Ala was utilized $\left(\mathrm{JR}_{2} \mathrm{ECref}_{2}\right)$. Although chemically identical to $\mathrm{JR}_{2} \mathrm{EC}_{2}$ this peptide can under no circumstances heteroassociate and fold. ${ }^{[4]}$ The UV-vis spectra 15 showed a slight decrease in extinction and a shift of the baseline but no red-shift of the plasmon bands could be seen, indicating that a certain amount of unspecific bridging aggregation may occur resulting in loosely associated aggregates (Figure $3 \mathrm{c}$ ). However, no changes in the positions of either the longitudinal or 20 the transverse plasmon peaks were observed over a period of 30 minutes (Figure 4a). TEM micrographs (Figure 4b) indicated formation of extensive aggregates after addition of $\mathrm{JR}_{2} \mathrm{EC}_{2}$ whereas only dispersed particles were observed after addition of $\mathrm{JR}^{2} \mathrm{EC}_{\text {ref2 }}$. The aggregation as well as the dependence of folding 25 on the aggregation was further confirmed using dynamic light scattering (Figure S4, Supporting Information). No aggregation was obtained using $\mathrm{JR}_{2} \mathrm{EC}_{\mathrm{ref} 2}$ whereas a gradual increase in aggregate size was seen upon addition of $\mathrm{JR}_{2} \mathrm{EC}_{2}$. The overgrowth procedure thus enables a homogenous thiol30 dependent and direct immobilization of JR2KC onto the Au AHNRs, which enabled a folding-mediated particle assembly as schematically outlined in Scheme 1.

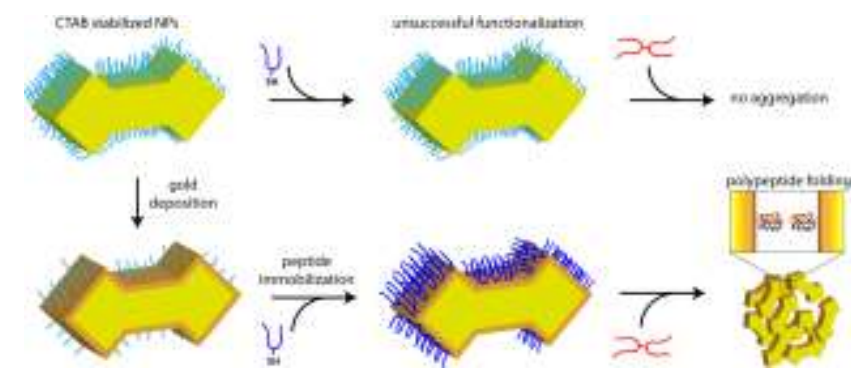

Scheme 1. Polypeptide immobilization and folding-mediated assembly.

In agreement with previous results on hetero-association and folding-mediated aggregation of spherical gold nanoparticles, ${ }^{[12 b]}$ 40 there was a critical concentration of the bridging polypeptide required before any aggregation was observed. For concentrations of $1-5 \mu \mathrm{M}$ of the bridging polypeptide extensive aggregation of the NRs was observed, whereas at lower concentrations no aggregation was obtained (Figure 5a,b). This is within the same 45 concentration range as for the assembly of spherical Au NPs using these polypeptides. Interestingly, the onset of aggregation was observed at slightly lower concentrations of $\mathrm{JR}_{2} \mathrm{EC}_{2}$ in the transverse plasmon band. The rate of change of the position of the extinction maximum, which correlates to the rate of aggregation,
50 was also larger for the transverse band than for the longitudinal band for all concentrations were aggregation was induced (Figure $5 \mathrm{c}, \mathrm{d})$.
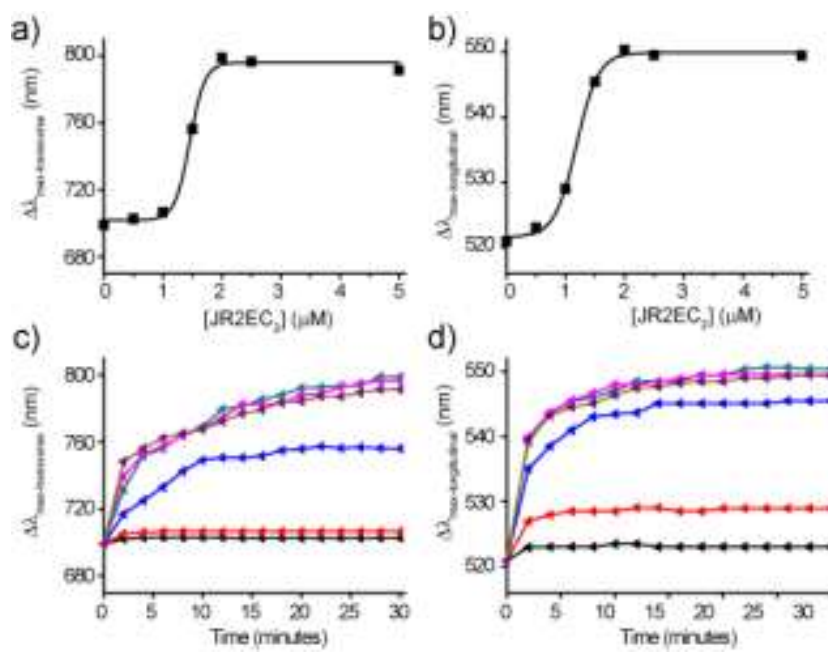

d)

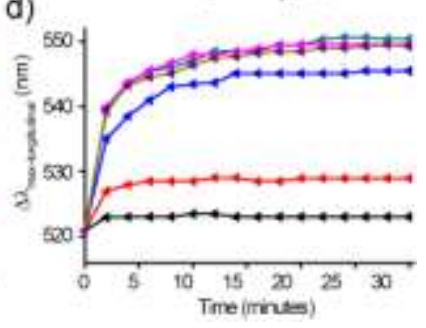

55 Figure 5. Position of the extinction maximum for the longitudinal (a) and transverse (b) plasmon peaks as a function of the concentration of JR2EC 2 . Change in the position of the extinction maximum over time for the longitudinal (c) and transverse (d) plasmon peaks for concentration of $\mathrm{JR}_{2} \mathrm{EC}_{2}$ of $0.5 \mu \mathrm{M}$ (black), $1 \mu \mathrm{M}$ (red), $1.5 \mu \mathrm{M}$ (blue), $2 \mu \mathrm{M}$ (green), 2.5 60 $\mu \mathrm{M}$ (pink) and $5 \mu \mathrm{M}$ (purple). a)

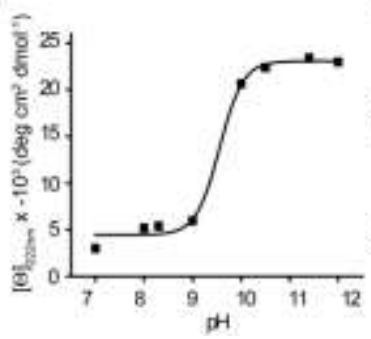

b)

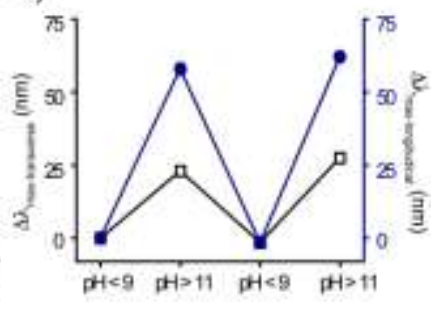

Figure 6. a) Mean residue molar ellipticity at $222 \mathrm{~nm}$ for JR2KC as a 65 function of $\mathrm{pH}$. b) $\mathrm{pH}$-induced changes in the position of the extinction maximum for the longitudinal (circles) and transverse (squares) plasmon peaks for the JR2KC functionalized NRs.

The reversibility of the peptide-mediated nanorod assembly 70 was confirmed using the pH-responsiveness of the JR2KC polypeptide. JR2KC homodimerize and fold at $\mathrm{pH} \sim 10$ due to the deprotonation of lysine residues at the dimerization interface which was confirmed using circular dichroism spectroscopy (Figure 6 a). Increasing the $\mathrm{pH}>11$ induced extensive 75 aggregation of the JR2KC functionalized nanorods (Figure $6 \mathrm{~b}$ ). The particles were redispersed completely when lowering the $\mathrm{pH}$ $<9$. The particles could be repeatedly assembled and redispersed.

\section{Conclusions}

80 The results obtained here are in excellent agreement with previous results on DNA-mediated assembly of CTAB stabilized gold $\mathrm{NRs}^{[8]}$ and strongly indicate that the presence of silver and 
silver ions indeed have a strong negative influence on the specific thiol-mediated immobilization of molecules on the particles. Since thiolated molecules binds strongly also to silver, the presence of $\operatorname{Ag}(0)$ is not likely to be the cause for these 5 observations. More likely is that the strongly bound CTAB and silver bromide complex interfere with the binding of the thiol to the gold surface. After the overgrowth procedure and during peptide immobilization the buffer still contained small amounts of CTAB $(0.1 \mathrm{mM})$, suggesting the presence of small amounts of 10 the surfactant has no obvious influence on the immobilization of the peptides. Here, loosely adsorbed CTAB may actually facilitate the functionalization of the particles by preventing unspecific bridging aggregation by the highly positively charged JR2KC $(+11$ at neutral $\mathrm{pH})$ at low peptide surface coverage, 15 which has been observed for citrate capped spherical Au NPs (unpublished data).

The employment of a simple overgrowth procedure that forms a thin gold film that covers adsorbed silver species can drastically improve the versatility of anisotropic nanoparticles since the 20 adsorbed CTAB can easily be replaced without compromising particle stability. Using this strategy, a de novo designed polypeptide was immobilized on arrowhead gold nanorods, which enabled a homogenous folding-mediated self-assembly of the particles. The ability to tune the surface chemistry of arrowhead 25 gold nanorods is a prerequisite to fully exploit these nanomaterials in applications such as biosensors, plasmonic devices and therapeutics. This also holds true for other types of CTAB stabilized anisotropic gold nanoparticles making this strategy highly interesting for the development of novel

30 plasmonic nanomaterials with specific and desirable chemical functionalities.

\section{Acknowledgements}

The authors are grateful for the financial support from the Knut 35 and Alice Wallenberg Foundation (KAW), the Swedish Research Council (VR), and the Swedish Foundation for Strategic Research (SSF).

\section{Notes and references}

$40{ }^{a}$ Centre for Biomimetic Sensor Science, Nanyang Technological University, 50 Nanyang Drive, Singapore 639798. E-mail:

bliedberg@ntu.edu.sg

${ }^{b}$ School of Materials Science and Engineering, Nanyang Technological University, 50 Nanyang Avenue, Singapore 639798.

$4{ }^{c}$ Division of Molecular Physics, Department of Physics, Chemistry and

Biology, Linköping University, 58183 Linköping, Sweden.

${ }^{d}$ Department of Biochemistry and Organic Chemistry, Uppsala

University, BMC, Box 576, 75123 Uppsala, Sweden.

$\dagger$ Electronic Supplementary Information (ESI) available: Electron

50 micrographs of NRs before and after gold overgrowth, and peptide mediated aggreation. See DOI: 10.1039/b000000x/

1 a) C. A. Mirkin, R. L. Letsinger, R. C. Mucic and J. J. Storhoff, Nature, 1996, 382, 607; b) A. P. Alivisatos, K. P. Johnsson, X. Peng, T. E. Wilson, C. J. Loweth, M. P. Bruchez Jr and P. G. Schultz,

55 Nature, 1996, 382, 609; c) W. Cheng, M. J. Campolongo, J. J. Cha, S. J. Tan, C. C. Umbach, D. A. Muller and D. Luo, Nat. Mat., 2009, 8, 519 .

2 S. J. Zhen, C. Z. Huang, J. Wang and Y. F. Li, J. Phys. Chem. C, 2009, 113, 21543
603 K. K. Caswell, J. N. Wilson, U. H. F. Bunz and C. J. Murphy, J. Am. Chem. Soc., 2003, 125, 13914.

4 D. Aili, K. Enander, J. Rydberg, I. Lundström, L. Baltzer and B. Liedberg, J. Am. Chem. Soc., 2006, 128, 2194.

5 a) B. Nikoobakht and M. A. El-Sayed, Langmuir, 2001, 17, 6368; b)

65 B. Nikoobakht and M. A. El-Sayed, Chem. Mater., 2003, 15, 1957.

6 A. M. Alkilany, P. K. Nagaria, C. R. Hexel, T. J. Shaw, C. J. Murphy and M. D. Wyatt, Small, 2009, 5, 701.

7 a) A. Gole and C. J. Murphy, Chem. Mater., 2005, 17, 1325; b) X. Huang, I. H. El-Sayed, W. Qian and M. A. El-Sayed, J. Am. Chem. Soc., 2006, 128, 2115.

8 M. R. Jones, R. J. Macfarlane, B. Lee, J. A. Zhang, K. L. Young, A. J. Senesi and C. A. Mirkin, Nat. Mater., 2010, 9, 913.

9 a) T. K. Sau and C. J. Murphy, Langmuir, 2004, 20, 6414; b) F. Hubert, F. Testard and O. Spalla, Langmuir, 2008, 24, 9219.

7510 M. Liu and P. Guyot-Sionnest, J. Phys. Chem. B, 2005, 109, 22192.

11 Y. Niidome, Y. Nakamura, K. Honda, Y. Akiyama, K. Nishioka, H. Kawasaki and N. Nakashima, Chem. Commun., 2009, 1754.

12 a) K. Enander, D. Aili, L. Baltzer, I. Lundstrom and B. Liedberg, Langmuir, 2005, 21, 2480; b) D. Aili, K. Enander, L. Baltzer and B. $80 \quad$ Liedberg, Nano Lett., 2008, 8, 2473.

13 Y. Xiang, X. Wu, D. Liu, L. Feng, K. Zhang, W. Chu, W. Zhou and S. Xie, J. Phys. Chem. C, 2008, 112, 3203.

14 D. Aili, F. I. Tai, K. Enander, L. Baltzer and B. Liedberg, Angew. Chem., Int. Ed., 2008, 47, 5554

8515 Z. Sun, W. Ni, Z. Yang, X. Kou, L. Li and J. Wang, Small, 2008, 4, 1287.

16 G. L. Ellman, Arch. Biochem. Biophys., 1959, 82, 70.

17 N. R. Jana, L. Gearheart and C. J. Murphy, Adv. Mater., 2001, 13, 1389.

90 


\section{Specific functionalization of CTAB stabilized anisotropic gold nanoparticles with polypeptides for folding-mediated self-assembly} Yusong Wang,,$^{a, b}$ Daniel Aili, ${ }^{a, c}$ Robert Selegård, ${ }^{c}$ Yeeyan Tay, ${ }^{b}$ Lars Baltzer, ${ }^{d}$ Hua Zhang, ${ }^{a, b}$
Bo Liedberg*a,c

${ }^{a}$ Centre for Biomimetic Sensor Science, Nanyang Technological University, 50 Nanyang Drive, Singapore 639798. ${ }^{b}$ School of Materials Science and Engineering, Nanyang Technological University, 50 Nanyang Avenue, Singapore 639798. Division of Molecular Physics, Department of Physics, Chemistry and Biology, Linköping University, 58183 Linköping, Sweden. ${ }^{d}$ Department of Biochemistry and Organic Chemistry, Uppsala University, BMC, Box 576, 75123 Uppsala, Sweden.

Peptide synthesis: The polypeptides JR2EC, (NAADLEKAIEALEKHLEAKGPCDAAQLEK QLEQAFEAFERAG), and JR2KC (NAADLKKAIKALKKHLKAKGPCDAAQLKKQLKQ AFKAFKRAG), were synthesized on a Pioneer automated peptide synthesizer (Applied Biosystems) using standard fluorenylmethoxycarbonyl (Fmoc) chemistry. The crude products were purified by reversed-phase HPLC on a semi-preparative HICHROM C-8 column and identified by MALDI-TOF mass spectrometry. In order to obtain JR2 $\mathrm{KC}_{2}$, lyophilized peptide monomers $(1 \mathrm{mM})$ were dissolved in $0.1 \mathrm{M}$ ammonium bicarbonate buffer $\mathrm{pH} 8$, aerated for 90 minutes and incubated at $4^{\circ} \mathrm{C}$ for at least 24 hours before use. Complete oxidation was confirmed using a standard Ellman's test for determination of free thiols. ${ }^{1}$

High resolution TEM:
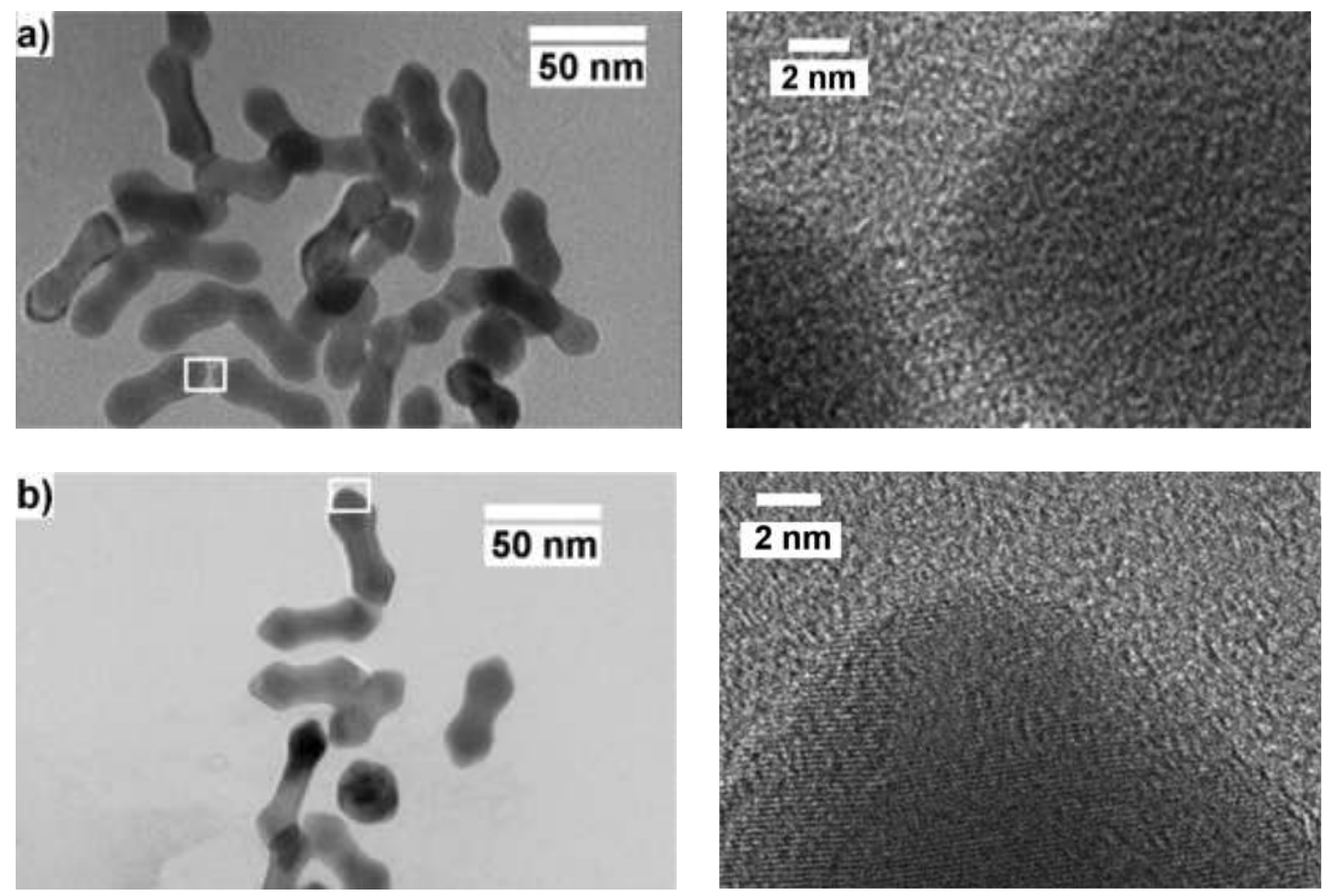

Figure S1. HR-TEM image of the arrowhead nanorods before (a) and after (b) Au layer growth. Insets are the enlarged area of the gold particles in a) as indicated by the white box. 
FT-IR: FT-IR spectra was recorded of soloution casted multilayers of JR2KC modifed nanorods on $\mathrm{CaF}_{2}$ windows. In addition to the extensive washing after peptide immobilization, two additional centrifugation steps were carried out and the buffer was replaced by ultrapure water in order to elliminate any contributions from non-immobilized peptides. Spectra were recorded using a Vertex 70 instrument (Bruker Corp.) with a $2 \mathrm{~cm}^{-1}$ resolution. A three-term Blackmann-Harris apodization function was applied to the interferograms before Fourier transformation.

The amide I ( $\mathrm{C}=\mathrm{O}$ stretching) and amide II (C-N-H stretching and bending) bands apperead at 1655 and $1544 \mathrm{~cm}^{-1}$, respectively, which is in excellent agreement with previously published data of the same peptide without the Cys residue $\left(1656\right.$ and $\left.1545 \mathrm{~cm}^{-1}\right) .^{2}$ Additional bands at 1578 and $1412 \mathrm{~cm}^{-1}$, most likely correspond to the assymetric $-\mathrm{C}=\mathrm{COO}^{-}$and symmetric $-\mathrm{CO}_{2}$ streching vibrations of remianing actetate buffer, respectively. ${ }^{3}$

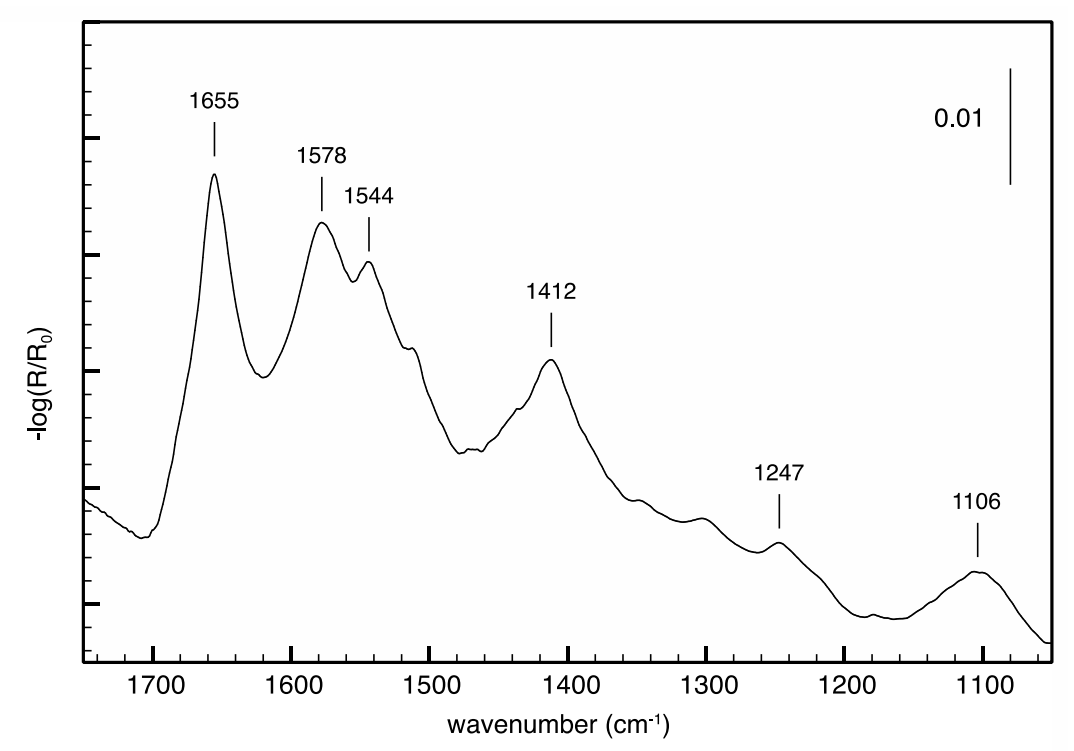

Figure S2. FT-IR spectrum of JR2KC modified gold nanorods. 


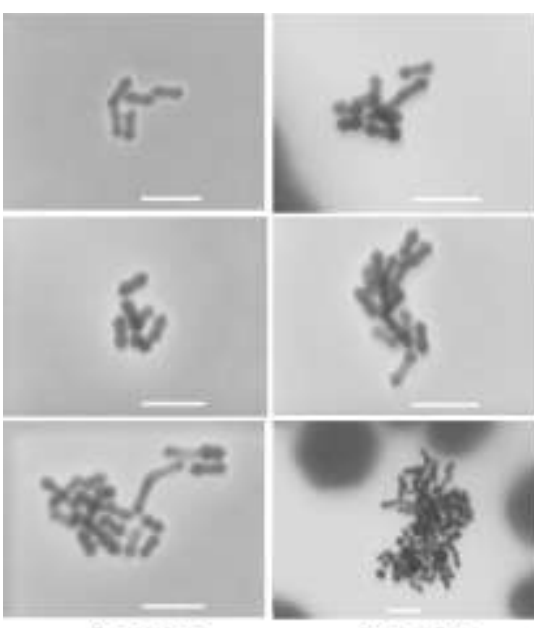

$2 \min$

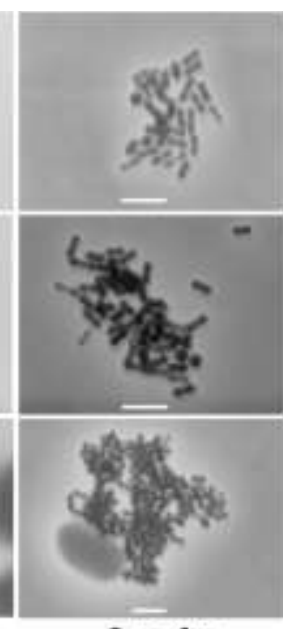

$6 \mathrm{~min}$

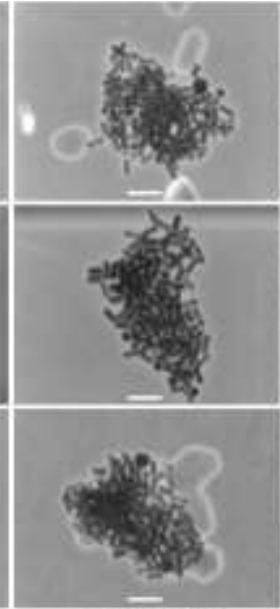

$8 \mathrm{~min}$

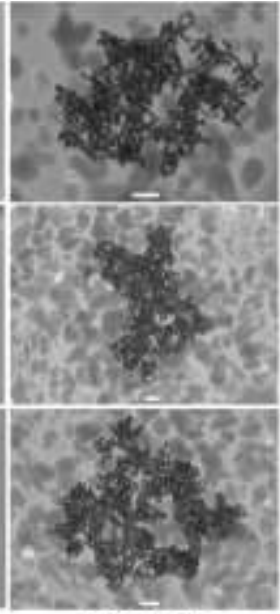

$16 \min$

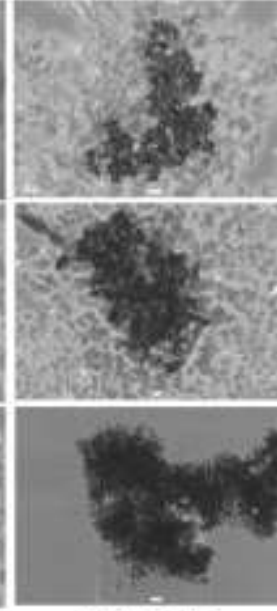

$30 \mathrm{~min}$

Figure S3. Representative FESEM-TED images of the assembly of JR2KC functionalized arrowhead gold nanorods after addition of $2 \mu \mathrm{M} \mathrm{JR} 2 \mathrm{EC}_{2}$ at different time points during the assembly in three different samples. Scale bars: $100 \mathrm{~nm}$.

Dynamic light scattering (DLS): DLS was carried out on a Malvern Nano Sizer using low volume cuvettes (ZEN0112). Typically, $18 \mu \mathrm{L}$ JR2KC functionalized NR stock solution ( $0.1 \mathrm{nM}$ ) was dispersed in $400 \mu \mathrm{L}$ fresh HBS-EP buffer (GE Healthcare, 0.01M HEPES, pH 7.4, $0.15 \mathrm{M} \mathrm{NaCl}, 3 \mathrm{mM}$ EDTA, 0.005\% surfactant P20) followed by DLS measurements. Peptides were added to the solution while mixing until the final polypeptide concentration was $\sim 2.3 \mu \mathrm{M}$. DLS was measured right after mixing as well as after 30 minutes. The same procedure was used for both $\mathrm{JR}_{2} \mathrm{EC}_{2}$ and $\mathrm{JR} 2 \mathrm{EC}_{\mathrm{ref} 2}$.

a)

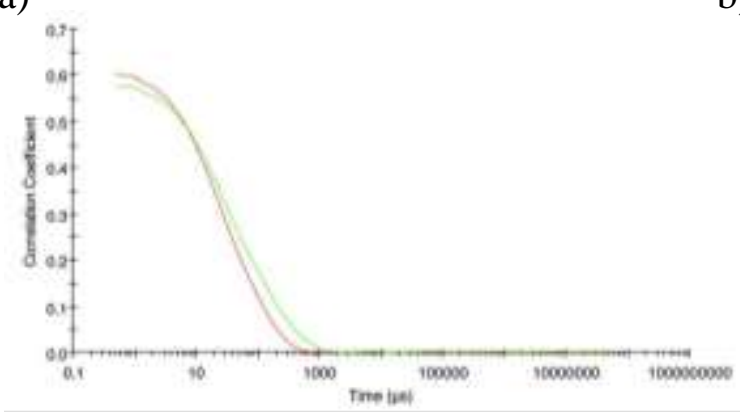

b)

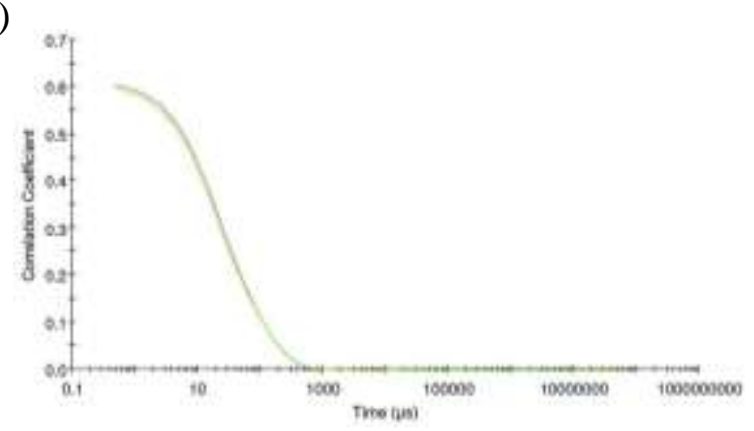


c)

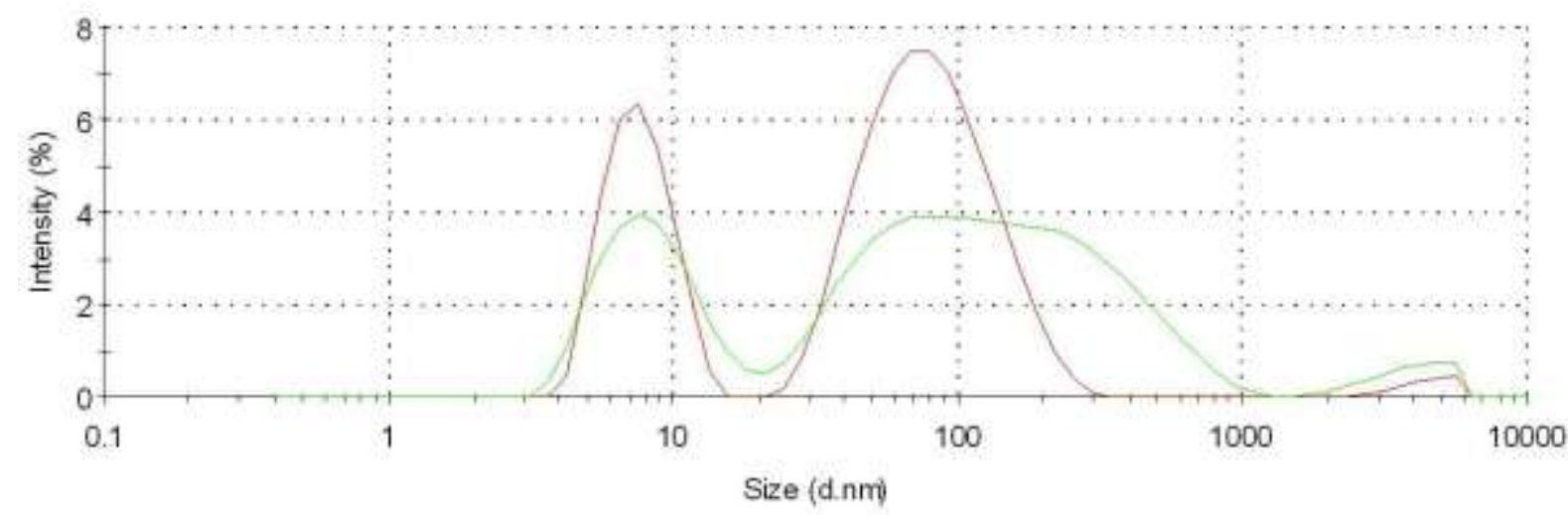

d)

Size Distribution by Intensity

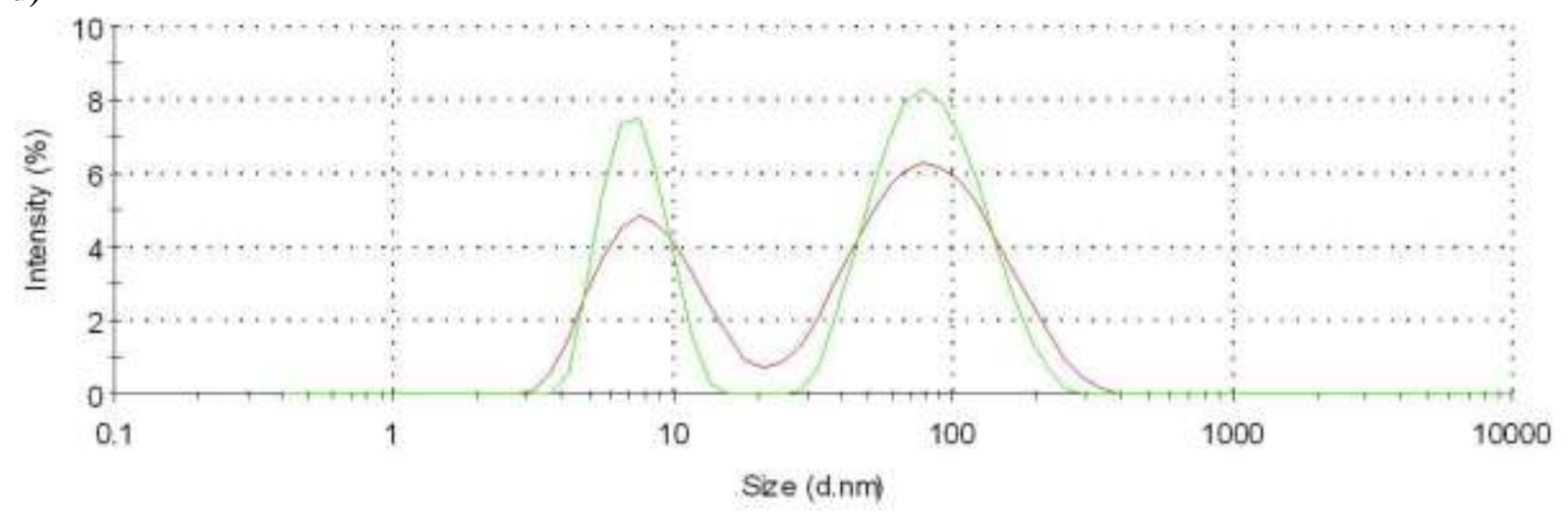

Figure S4. Correlation functions before (red) and after (green) addition of $\mathrm{JR}_{2} \mathrm{EC}_{2}$ (b) and $\mathrm{JR}_{2} \mathrm{EC}_{\mathrm{ref} 2}$ (b) and corresponding size distribution for JR2EC 2 (c) and JR2EC $\mathrm{Jef}_{2}$ (d).

\section{References:}

1. G. L. Ellman, Arch. Biochem. Biophys., 1959, 82, 70-77.

2. K. Enander, D. Aili, L. Baltzer, I. Lundström, B. Liedberg, Langmuir 2005, 21, 24802487.

3. J.-J. Max, C. Chapados, J. Phys. Chem. A, 2004, 108, 3324-3337. 